\title{
Inconsistency of the Wolf sunspot number series around 1848 (Research Note)
}

\author{
Raisa Leussu ${ }^{1,2}$, Ilya G. Usoskin ${ }^{1,2}$, Rainer Arlt ${ }^{3}$, and Kalevi Mursula ${ }^{1}$ \\ 1 Department of Physics, PO Box 3000, University of Oulu, 90014 Oulu, Finland \\ e-mail: Ilya.Usoskin@oulu.fi \\ 2 Sodankylä Geophysical Observatory (Oulu unit), University of Oulu, 90014 Oulu, Finland \\ ${ }^{3}$ Leibniz Institute for Astrophysics Potsdam, An der Sternwarte 16, 14482 Potsdam, Germany
}

Received 26 July 2013 / Accepted 23 September 2013

\begin{abstract}
Aims. Sunspot numbers form a benchmark series in many studies, but may still contain inhomogeneities and inconsistencies. In particular, an essential discrepancy exists between the two main sunspot number series, Wolf and group sunspot numbers (WSN and GSN, respectively), before 1848. The source of this discrepancy has remained unresolved so far. However, the recently digitized series of solar observations in 1825-1867 by Samuel Heinrich Schwabe, who was the primary observer of the WSN before 1848, makes such an assessment possible.

Methods. We construct sunspot series, similar to WSN and GSN but using only Schwabe's data. These series, called here WSN-S and GSN-S, respectively, were compared with the original WSN and GSN series for the period 1835-1867 for possible inhomogeneities. Results. This study supports the earlier conclusions that the GSN series is more consistent and homogeneous in the earlier part than the WSN series. We show that: the GSN series is homogeneous and consistent with the Schwabe data throughout the entire studied period; the WSN series decreases by roughly $20 \%$ around 1848 , which is caused by the change of the primary observer from Schwabe to Wolf and an inappropriate individual correction factor used for Schwabe in the WSN; this implies a major inhomogeneity in the WSN, which needs to be corrected by reducing its values by $20 \%$ before 1848 ; the corrected WSN series is in good agreement with the GSN series.
\end{abstract}

Key words. Sun: activity - sunspots - planetary systems

\section{Introduction}

Sunspot numbers form the longest series of direct astronomical observations. Beginning in 1610, systematic counts and drawings of sunspots have been carried out through centuries by an army of professionals and amateurs (for reviews, see, Hathaway 2010; Usoskin 2013). The tremendous work by Rudolf Wolf of Zürich in the second half of the 19th century resulted in creation of the first official sunspot number series, known as the Wolf sunspot numbers (WSN). The core of the WSN series is based on the observations made by Rudolf Wolf and his successors after 1848 and have continued to present times. However, the quality of the WSN series before 1848 is sometimes doubted as it is a result of compilation by Wolf of different observations and records and includes poorly grounded re-calibration (e.g. Sonett 1983; Wilson 1988, 1998; Usoskin 2013). Another series of sunspot activity, known as the group sunspot numbers (GSN) was compiled much more recently (Hoyt \& Schatten 1998). It is methodologically different from the WSN and contains a much greater amount of original information than the WSN and is often considered to be more homogeneous and representative in the early part of the series than the WSN (e.g., Hathaway et al. 2002; Usoskin 2013).

Since the sunspot numbers form a benchmark series for many research topics and practical applications ranging from statistical analysis to solar and terrestrial physics, it is crucially important to verify the homogeneity of the sunspot number series, particularly on the long timescale. However, it has been difficult to directly test the quality of the two sunspot number series or their relative correctness in the earlier part (e.g., Wilson 1998) until now.

Thanks to the recent work by Arlt et al. (2013), all individual drawings of one of the most famous and scrupulous solar observers Samuel Heinrich Schwabe, in 1825-1867 have now been digitized, forming an independent homogeneous series of sunspot observations. Accordingly, we use this new data based on Schwabe's observations to study the consistency of the WSN and GSN series for the period between 1835 and 1867.

\section{Sunspot number series}

\subsection{Wolf sunspot numbers WSN}

The Wolf sunspot number, also known as the Zürich sunspot numbers series, is calculated as

$\mathrm{WSN}=k(10 G+N)$,

where $G$ and $N$ are the number of sunspot groups and the number of individual sunspots reported by the selected observer for a given day, respectively, and $k$ is the observer's correction factor which accounts for his experience and the quality of his instrument. The WSN uses only one observation per day by the primary observer as selected by Wolf and his successors. If the primary observer did not report solar observations for a particular day, secondary, tertiary etc., observers are used. The hierarchical system of observers (Waldmeier 1961) was established with the purpose of homogeneity of the series. This system of the WSN 


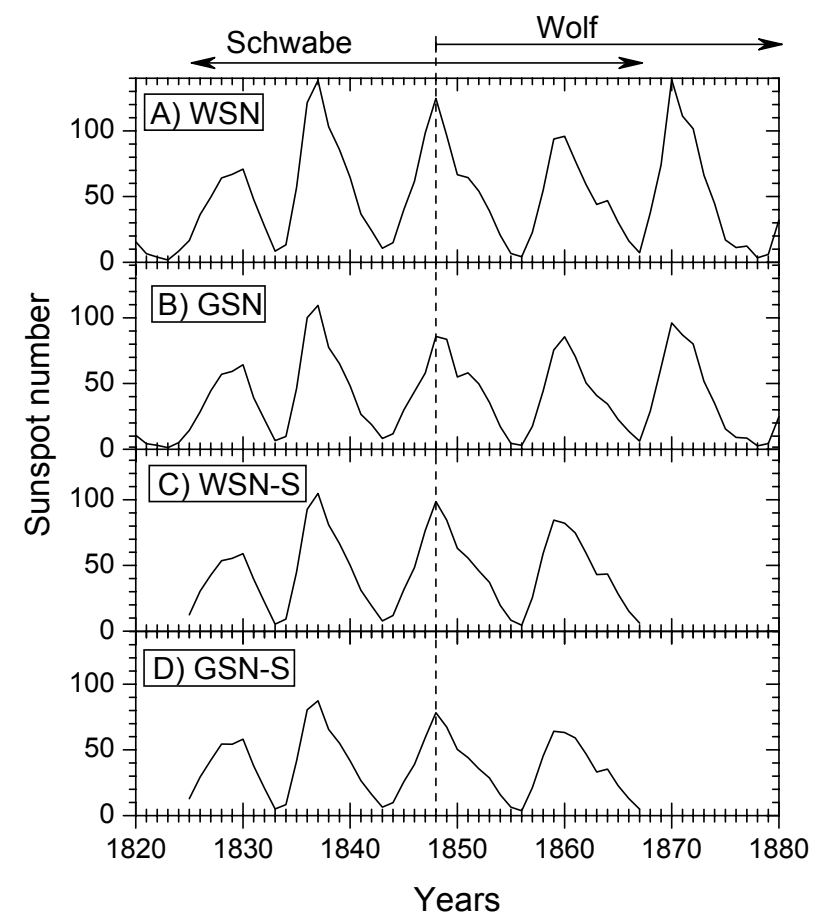

Fig. 1. Annual sunspot numbers: a) the official Wolf sunspot number WSN; b) the group sunspot number GSN; c) the WSN calculated from Schwabe data, WSN-S; d) the GSN calculated from Schwabe data, GSN-S. Periods of sunspot observations by Schwabe and Wolf are indicated at the top.

was changed in the late 20th century for the international sunspot number (Clette et al. 2007) but was still used during the period under investigation. It is important that Schwabe was the primary observer for the Wolf series for the period 1826 through 1847. In 1848-1893 Wolf himself was the primary observer. Thus, the period studied here is characterized by two primary observers Schwabe before 1848 and Wolf after that. The WSN series for the period under question is shown in Fig. 1a.

\subsection{Group sunspot numbers GSN}

The group sunspot number, which was introduced by Hoyt \& Schatten (1998), is based only on the number of sunspot groups without individual spots. The GSN is supposed to be more homogeneous in the long term than the WSN since the sunspot groups are more robustly defined than individual spots. Another principal difference between the GSN and WSN is that the former uses all the available observations for a given day and not only the one by the primary observer. The definition of the GSN is

$\mathrm{GSN}=\frac{12.08}{n} \sum_{i=1}^{n} k_{i} G_{i}$,

where $G_{i}$ is the number of sunspot groups as reported by the $i$ th observer, $k_{i}$ is the individual correction factor of the observer, and $n$ is the number of observers whose data are available for the given day. Factor 12.08 is included to normalize the GSN to the same absolute level as the WSN in 1874-1976 (Hoyt \& Schatten 1998). The GSN series is usually considered as more homogeneous in its earlier part than the WSN (Letfus 1999; Hathaway et al. 2002; Usoskin \& Kovaltsov 2004). The GSN series around the period under question is shown in Fig. $1 b$.

\subsection{Schwabe sunspot numbers WSN-S and GSN-S}

Samuel Heinrich Schwabe was an amateur astronomer, who performed daily observations of the Sun at his home in Dessau, Germany, nearly continuously from 1825 until 1868. His sunspot observations contain over 8000 drawings of the solar disk and over 3000 verbal descriptions. These data, which include drawings, have been recently digitized and tabulated, as reported by Arlt et al. (2013). The data set produced from these measurements contain information on the date, heliospheric coordinates and subjective size of each individual spot drawn by Schwabe. The daily numbers of groups and spots were calculated from these data to be compared with the official indices for this period of time.

In addition to drawings, Schwabe also defined sunspot groups for each day. Rudolf Wolf, who used Schwabe as the primary observer from 1826 to 1847 , listed the number of spots and groups for almost each day from Schwabe notes. To estimate the relation between Schwabe's group definition and the one Rudolf Wolf presumably had, we compared Schwabe's groups to those defined by Rudolf Wolf during the same time period (Wolf 1850). Out of all the 8401 days when comparison is possible, there were only 19 cases when Schwabe had assigned one group more than Wolf, and 25 cases, when it was one group less. Considering the small number $(0.5 \%)$ of differing assignments, it is obvious that Wolf and Schwabe had a very similar perception on the definition of sunspot groups, and these assignments can be used in further calculations. We note that their definition is slightly different from the modern definition of sunspot groups, but this does not affect the results, since the Schwabe and Wolf numbers are compared to each other and not calibrated with today's values.

Using Schwabe's definition of the groups and individual spots, we computed analogs of the WSN and GSN series based solely on Schwabe's data. The daily WSN-S and GSN-S were calculated by means of Eqs. (1) and (2), respectively, using the daily numbers of spots and groups defined in Schwabe's data. The correction factors $k$ were set to unity. The monthly averages were acquired by calculating mean values of WSN-S and GSN-S from the daily data, and the annual averages by calculating mean values of the monthly data. The resulting annual means of WSN-S and GSN-S are shown in Fig. 1c and d.

We note that the accuracy of Schwabe's observations gradually changed during the first ten years of his observations most likely because of the changing drawing style. For example this can be observed from Fig. 2, where the mean size of spots drawn by Schwabe is shown as function of time. During 1826-1835, he mostly plotted large spots, while he also recorded spots of smaller size homogeneously after ca. 1835. For whatever reason, this suggests that many small spots might have been left unnoticed before 1835 . This consequently reduces the WSN values compared to GSN at this time, since the number of sunspot groups can be identified more robustly. Therefore, we limit ourselves to the period of 1835-1867, when Schwabe's series is homogeneous, for the present analysis.

\section{Comparing different sunspot numbers}

Here, we compare the (annual values of) sunspot series as defined in Sect. 2, by analyzing their ratios to get rid of the scaling ambiguity. To avoid large uncertainties related to division of small numbers, we omitted the years when the corresponding annual sunspot number in either series is smaller than 10 . The ratios are shown in Fig. 3. 
Table 1. Mean ratios, along with standard error, of different annual series (see Fig. 3) before and after 1848, for their difference $D$ and for the significance level $s$ at which the null hypothesis of the constant ratio can be rejected.

\begin{tabular}{lcccc}
\hline \hline Data & WSN/GSN & WSN-S/GSN-S & WSN/WSN-S & GSN/GSN-S \\
\hline $1835-1848$ & $1.35 \pm 0.04$ & $1.21 \pm 0.02$ & $1.27 \pm 0.01$ & $1.14 \pm 0.03$ \\
$1849-1867$ & $1.19 \pm 0.02$ & $1.26 \pm 0.01$ & $1.06 \pm 0.02$ & $1.14 \pm 0.04$ \\
$D$ & $0.16 \pm 0.045$ & $0.05 \pm 0.022$ & $0.21 \pm 0.022$ & $0.0 \pm 0.05$ \\
$S$ & $5 \times 10^{-4}$ & 0.03 & $<10^{-7}$ & 0.96 \\
\hline
\end{tabular}

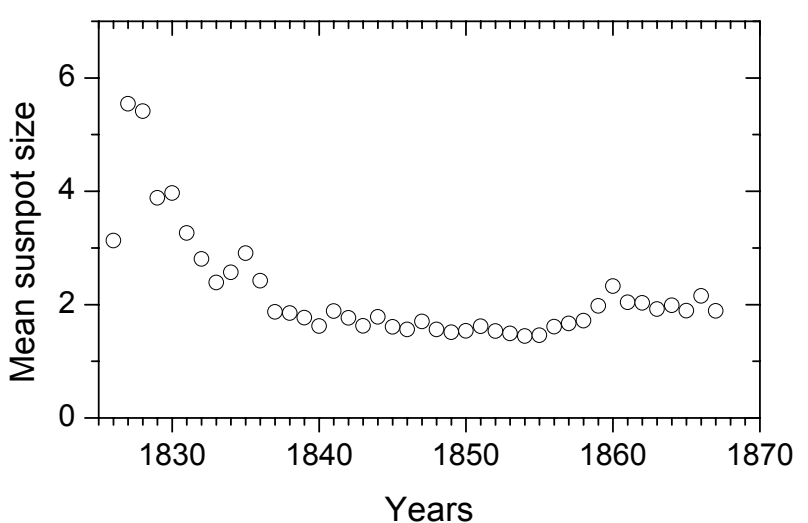

Fig. 2. Mean size (in arbitrary units used during the digitization) of sunspots drawn by Schwabe.

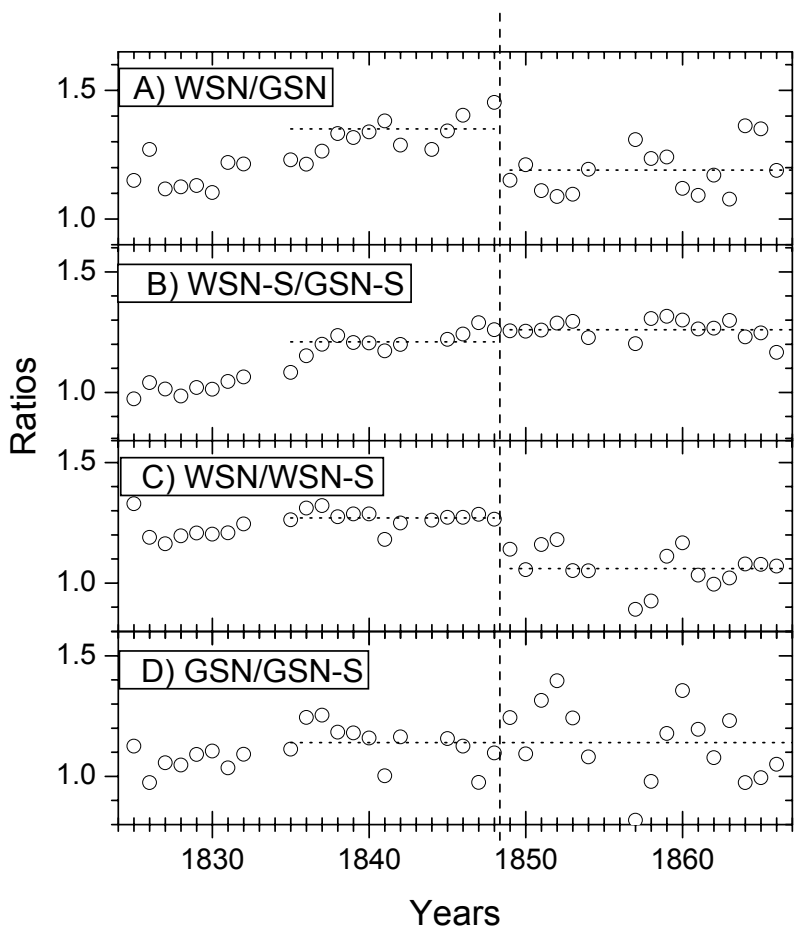

Fig. 3. Ratios of the annual sunspot numbers: a) the ratio of WSN to GSN; b) the ratio of WSN-S to GSN-S; c) the ratio of WSN to WSN-S; d) the ratio of GSN to GSN-S. The vertical dashed line indicates the period when R. Wolf started his observations. Horizontal dotted lines depict the mean ratios before and after that date. Details of statistics are collected in Table 1 .

\subsection{WSN vs. GSN}

First, we compare the two official series, WSN and GSN. The ratio of the official Wolf sunspot numbers and the official group sunspot numbers is shown in Fig. 3a. A jump in the ratio is apparent around 1848, when the primary observer of the
WSN series had changed from Schwabe to Wolf. The jump from 1.35 to 1.19 is significant at the level of $5 \times 10^{-4}$ (see Table 1 , Col. 2). This indicates that the WSN and GSN series are mutually inconsistent but cannot verify the consistency of either series.

\subsection{WSN-S vs. GSN-S}

Next, we analyze the ratio of WSN-S to GSN-S (Fig. 3b and third column in Table 1). Both series are based solely on Schwabe's data and thus their ratio is expected to be stable. One can see, however, that there is a small difference likely related to the detection of small spots by Schwabe in the early part of the period, as discussed in Sect. 2.3 (Fig. 2). Otherwise, the ratio is perfectly smooth with a small tendency to decrease slightly around solar minima, which is probably because of the smaller size of spots.

\section{3. $W S N$ vs. WSN-S}

The ratio between WSN and WSN-S is shown in Fig. 3c and in the fourth column in Table 1. A sudden and highly significant jump is apparent at 1848-1849, exactly at the time when the primary observer of the WSN series switched from Schwabe to Wolf. This suggests that the WSN series suffers a calibration problem in the middle of the 19th century. The jump is highly significant (see Table 1).

\subsection{GSN vs. GSN-S}

The ratio of GSN to GSN-S is shown in Fig. 3d and in the fifth column in Table 1. It appears nearly constant throughout the entire period, confirming the homogeneity of the GSN series in the studied time interval.

\section{Discussion}

Let us now summarize the results from comparing the four sunspot series, where two are official ones and the other two are based on Schwabe's observations. The latter are perfectly homogeneous (at least after 1835 - see Sect. 2.3), being based on the systematic observations performed by an experienced observer using the same instrumentation and techniques. This makes it possible to check the official series for homogeneity and internal consistency for the middle 19th century.

The comparison shows that the GSN series is homogeneous around 1848, since the ratio between the official GSN series, which involve data from many observers, and the GSN-S series, which based solely on Schwabe's data, is constant throughout the studied time interval. On the other hand, the WSN series appears inconsistent and experiences a significant decrease around 1848. This decrease is observed relative to both the official GSN series (by 14\%) and WSN-S (by 20\%). The latter value suggests that the individual correction factor $k$ (see Eq. (1)) used by R. Wolf for Schwabe was inappropriate and that it should be 


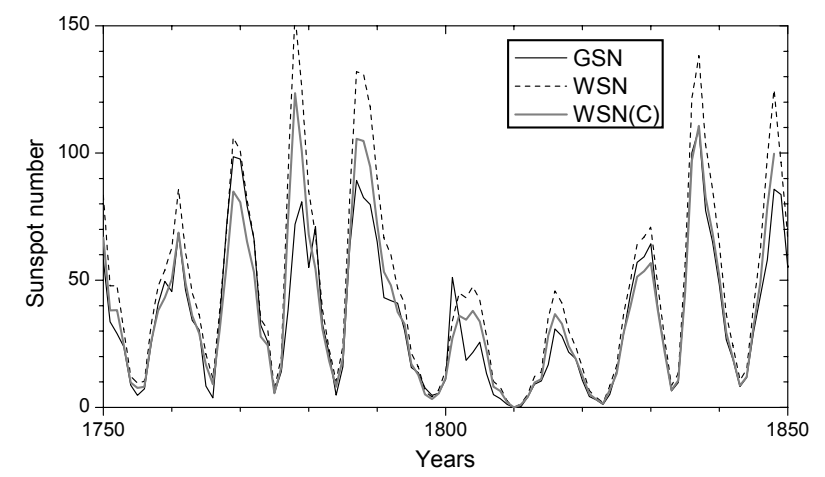

Fig. 4. Annual sunspot numbers for the period 1750-1850 AD: the official GSN series (solid GSN line), the official WSN series (dashed WSN line) and the WSN series that is $20 \%$ reduced (grey WSN-C line).

lowered by $20 \%$ to make the WSN series homogeneous through the change of the primary observer in 1848 . This requires the corresponding reduction of the WSN values by $20 \%$ for the period 1826-1848 when Schwabe was the primary observer for WSN. Moreover, this leads to the $20 \%$ reduction of the entire WSN series before 1848 since the "calibration" of the WSN series is consecutive in time using overlaps between observers. The WSN series corrected for this $20 \%$ reduction is shown as the WSN-C series in Fig. 4 along with the official WSN and GSN series. One can see that the corrected WSN is in a much better agreement with the GSN than the official WSN series. We note that the main reason for this scatter in the ratios between these series is most likely related to the change of observers and not to some intrinsic variation in the qualities of sunspots and their distribution. It should also be noted that the WSN might also need some correction for the period 1826-1835, as discussed earlier.

\section{Conclusions}

In this paper we have compared the official Wolf sunspot number and the group sunspot number series with the homogeneous multidecadal record (1835-1867) of sunspot observations by Samuel Heinrich Schwabe, which have been recently digitized by Arlt et al. (2013). We show the following:

- The GSN is homogeneous and consistent with Schwabe's data throughout the entire studied period.
- The WSN series suffers a significant $20 \%$ decrease around 1848 , which is caused by the switch in the primary observer from Schwabe to Wolf.

- The decrease reflects an inappropriate individual correction factor used for Schwabe in the WSN and implies a major inhomogeneity in the WSN.

- The WSN needs to be corrected by decreasing its values by $20 \%$ before 1848 .

- The so corrected WSN series agree well with the GSN series.

- Before 1835, the WSN might be underestimated because Schwabe only considered large spots. A detailed study will be made later.

This study supports the earlier conclusions that the group sunspot number series (Hoyt \& Schatten 1998) is more consistent and homogeneous than the Wolf sunspot number series in earlier observations (e.g., Letfus 1999, 2000; Hathaway et al. 2002; Usoskin \& Mursula 2003; Usoskin 2013).

Acknowledgements. The data for the official monthly Wolf sunspot number is from SIDC and the official monthly group sunspot number data from NOAA. R.L. acknowledges support from the Väisälä Foundation through the Finnish Academy of Science and Letters. The authors would like to acknowledge networking support by COST Action ES1005 "TOSCA" (http://www . tosca-cost.eu/).

\section{References}

Arlt, R., Leussu, R., Giese, N., Mursula, K., \& Usoskin, I. G. 2013, MNRAS, 433, 3165

Clette, F., Berghmans, D., Vanlommel, P., et al. 2007, Adv. Space Res., 40, 919 Hathaway, D. H. 2010, Liv. Rev. Sol. Phys., 7, 1

Hathaway, D. H., Wilson, R. M., \& Reichmann, E. J. 2002, Sol. Phys., 211, 357

Hoyt, D. V., \& Schatten, K. H. 1998, Sol. Phys., 181, 491

Letfus, V. 1999, Sol. Phys., 184, 201

Letfus, V. 2000, Sol. Phys., 194, 175

Sonett, C. P. 1983, J. Geophys. Res., 88, 3225

Usoskin, I. G. 2013, Liv. Rev. Sol. Phys., 10, 1

Usoskin, I. G., \& Kovaltsov, G. A. 2004, Sol. Phys., 224, 37

Usoskin, I. G., \& Mursula, K. 2003, Sol. Phys., 218, 319

Waldmeier, M. 1961, The sunspot-activity in the years 1610-1960 (Zurich: Schulthess)

Wilson, R. M. 1988, Sol. Phys., 117, 269

Wilson, R. M. 1998, Sol. Phys., 182, 217

Wolf, R. 1850, Astronomische Mitteilungen der Eidgenössischen Sternwarte Zürich, 1, 247 